\title{
Supplemental Methodology
}

\section{Human patients}

Pregnant women who had decided on termination of pregnancy due to maternal anxiety provided consents. Normal fetal pancreata were collected from aborted fetuses of the first and second trimesters at gestational weeks 11.3 and 24.6, respectively. In addition, adult pancreatic biopsies were collected from patients with pancreatic cysts or gastrointestinal cancers as reported previously (13). All procedures were approved by The Chinese University of Hong Kong Hospital Authority (NTEC) Joint Clinical Research Ethics Committee.

\section{Mice}

The $P d x 1^{C r e E R T /+} ; Y Y 1^{f l f l}$ mouse line was generated by crossing $P d x 1^{C r e E R T}$ (a gift from Professor Douglas Melton, Harvard University (14)) with $Y Y 1^{f l f l}$. $Y Y 1^{f l f l}$ was described previously (15). To induce $Y y 1$ ablation in $P d x 1^{C r e E R T /{ }^{+}} ; Y Y 1^{f l f l}$, tamoxifen (Sigma) was dissolved in corn oil (10 $\mathrm{mg} / \mathrm{mL}$ ) and eight doses with each at $100 \mathrm{ug} / \mathrm{g}$ body weight was given to 6-8 weeks old adults within two weeks or two doses with each at $100 \mathrm{ug}$ were given to neonates at P1 and P3 through intraperitoneal (i.p.) injection. All mice used in physiological tests were male. All of the procedures were approved by the CUHK Animal Experimentation Ethics Committee.

\section{Cell cultures}

The rat Ins-1 beta cell line was cultured in RPMI1640 supplemented with 10\% FBS, 1\% penicillinstreptomycin (Pen-Strep) and $0.05 \mathrm{mM}$ 2-mercaptoethanol (2-ME). The mouse MIN6 beta cell line was cultured in DMEM supplemented with 15\% FBS, 1\% Pen-Strep and 0.05 mM 2-ME. Transient transfections of cells with siRNA (GenePharma) against YY1 (5'GAAGAUGAUGCUCCAAGAATT3' and 5'UUCUUGGAGCAUCAUCUUCTT3') or scrambled control (5, UUCUCCGAACGUGUCACGUTT3' and 5'ACGUGACACGUUCGGAGAATT3') were performed using RNAimax (Thermo Fisher Scientific) per manufacturer's instructions, and transfections with DNA plasmid encoding YY1 
were performed using Lipofectamine 3000 (Thermo Fisher Scientific) as described previously (15). Stable knockdown was achieved by cloning short hairpin (sh)RNA against YY1 (target squence:5'ACATCTTAACACACGCTAAAGTTCAAGAGACTTTAGCGTGTGTTAAGATGT

TTTTTT3') or against scrambled shRNA (target sequence: 5' TTCTCCGAACGTGTCACGTTTCAAGAGAACGTGACACGTTCGGAGAATTTTTT3’)

along with the pLenti-U6 packaging plasmid (Vigene Biosciences). For luciferase experiments, the enhancer regions with or without (mutant) the YY1 core motif sequence were cloned into the pGL3 luciferase reporter vector (Promega). Cells were transfected with DNA plasmid encoding YY1 or empty vector in 24 well plates. Cell extracts were prepared and luciferase activity was monitored using the Dual-Luciferase Reporter Assay System (Promega) as previously described (15).

\section{Blood glucose monitoring, glucose tolerance and insulin tolerance tests}

The fed blood glucose levels were monitored regularly at the same time every day, and the fasting blood glucose levels were measured after an overnight fasting for 14 - 16 hours. Tail vein blood samples were collected and blood glucose levels were measured by a glucometer (Contour ${ }^{\circledR}$ TS, $^{2}$ Bayer). For glucose tolerance test and in vivo glucose stimulated insulin secretion (GSIS) assay, mice were i.p. injected with glucose ( $2 \mathrm{mg} / \mathrm{g}$ body weight) after $14-16$ hour fasting, and the blood glucose and insulin levels were respectively monitored at specific time points after glucose injection. Blood insulin levels were measured by the mouse ultrasensitive insulin ELISA kit (ALPCO Diagnostics, Cat. No. 80-INSMSU-E01). For insulin tolerance test, mice were i.p. injected with human recombinant insulin $(0.5 \mathrm{U} / \mathrm{kg}$ body weight, Novo Nordisk) after 6 hour fasting, and the blood glucose levels were monitored at specific time points after insulin injection. The blood glucose or insulin readings were plotted against time after glucose or insulin injection, and the area under curve (AUC) was calculated using GraphPad Prism.

\section{Islet isolation}

Murine pancreatic islets were isolated by perfusion of the pancreata with Collagenase P (Roche, Cat. No. 11213865001) through the common hepatic bile duct at a concentration of $1 \mathrm{mg} / \mathrm{ml}$ of 
HBSS (Invitrogen). Pancreata were removed and dissociated at $37^{\circ} \mathrm{C}$ with shaking for 15 minutes. After several washes using RPMI1640 medium supplemented with 10\% FBS (Gemini Bio Products), islets were separated onto a gradient using a combination of serum-free RPMI1640 medium and Histopaque (Sigma-Aldrich, Cat. No. 10771). Islets were then hand-picked to avoid exocrine contamination and processed for different applications. For evaluation of islet insulin content, insulin was extracted by acid ethanol and islet lysates were measured by the mouse ultrasensitive insulin ELISA kit. For evaluation of islet proinsulin level, islet lysates were measured by the mouse proinsulin ELISA kit (Novus Biologicals, NBP2-69821).

\section{Ex vivo GSIS assays}

Briefly, approximately 10 size-matched pancreatic islets per group were rinsed twice with Krebs buffer and then preincubated in low $(2.8 \mathrm{mM})$ glucose Krebs for 2 hours at $37^{\circ} \mathrm{C}$ to remove residual insulin. Islets were then washed twice with Krebs, incubated in low glucose Krebs for 60 min, and supernatant was collected. After that, islets were washed twice in Krebs and incubated in high (16.7 mM) glucose Krebs for 60 min and supernatants were collected. Supernatants containing insulin were filtered through a $0.22 \mu \mathrm{m}$ filter before performing analysis using the mouse ultrasensitive insulin ELISA kit.

\section{Immunohistochemistry}

Human or murine pancreata were fixed in $4 \%$ paraformaldehyde at $4{ }^{\circ} \mathrm{C}$ overnight. The fixed tissues were washed three times with PBS and equilibrated in $30 \%$ sucrose at $4^{\circ} \mathrm{C}$ for 2 days before freezing and cryosectioning. Seven micrometer frozen sections were prepared. Samples were blocked with 5\% BSA and 5\% goat serum and then stained with the respective primary antibodies at $10 \mathrm{ug} / \mathrm{ml}$ at $4^{\circ} \mathrm{C}$ overnight. Anti-human primary antibodies used: YY1 (Abcam, Cat. No. ab109237), C-Peptide (DSHB, Cat. No. GN-ID4); or anti-mouse primary antibodies used: YY1 (abcam, Cat. No. 109237), INS (Dako, Cat. No. A0564), GCG (Abcam, Cat. No. ab82270), SST (Santa Cruz, Cat. No. sc-7819), cCASP3 (Cell Signaling Technology, Cat. No. 9661), and phospho- $\square$ H2A.X Ser139 (Cell Signaling Technology, Cat. No. 9718S). Alexa-Fluor-488- or Alexa-Fluor-546-conjugated secondary antibodies (Invitrogen) were used at room temperature for 
30 minutes in the dark. Slides were mounted with DAPI-containing fluorescence mounting medium (Dako) and fluorescence was detected with an upright fluorescence microscope, inverted fluorescence microscope or confocal microscope (all Leica). Images were processed with the ImageJ software (National Institutes of Health). Beta cell mass was analyzed based on this formula: $\mathrm{INS}^{+}$area/total pancreas area $\mathrm{x}$ pancreas weight, while beta cell size was calculated by $\mathrm{INS}^{+}$ area/ $/ \mathrm{NS}^{+}$nuclei.

\section{Immunofluorescence}

For evaluation of mitochondrial activity, isolated islets were digested into single cells through incubation in $0.25 \%$ trypsin at $37^{\circ} \mathrm{C}$ for 5 minutes. Chamber slides were pre-coated with $0.1 \mathrm{mg} / \mathrm{ml}$ poly-D-Lysine (Gibco, A38904-01) overnight followed by washing in PBS for three times before use. Individual cells were plated onto poly-D-Lysine coated chamber slides and cultured in RPMI1640 supplemented with 10\% FBS and 1\% Pen-Strep. The medium was carefully removed on the next day, immunostaining for MitoSOX Red indicator (ThermoFisher Scientific, Cat. No. M36008) based mitochondrial superoxide and tetramethylrhodamine ethyl ester (TMRE-PE, Cayman chemical, Cat. No. 701310) based mitochondrial membrane potential were respectively performed per manufacturers' instructions. After that, cells were fixed at $4 \%$ paraformaldehyde, followed by washing with PBS and blocking with 5\% BSA and 5\% goat serum. Cells were then stained with $10 \mathrm{ug} / \mathrm{ml}$ anti-mouse insulin antibody at $4^{\circ} \mathrm{C}$ overnight, followed by washing and staining with Alexa-Fluor-488 conjugated secondary antibody. Slides were then mounted with DAPI-containing fluorescence mounting medium and fluorescence was detected with a confocal microscope (Leica). Images were processed with the ImageJ software.

\section{Flow cytometry}

The isolated islets were dissociated into single cells through incubation in $0.25 \%$ trypsin at $37^{\circ} \mathrm{C}$ for 5 minutes. Single cells were blocked with $2 \%$ FBS followed by staining with fluorescence conjugated anti-mouse antibodies targeting CD3-APC/Cyanine 7 (Biolegend, 100221), CD4-PE (Biolegend, 100511), CD8a-APC (Biolegend, 100711), CD11b-PE (Biolegend, 101207), CD45APC/Cyanine 7 (Biolegend, 103116), F4/80-FITC (Biolegend, 123108), Ly6G-APC (Biolegend, 
127613), TMRE-PE or isotype controls for 30 minutes on ice. Cells were then washed thrice with FACS buffer, and DAPI (BD Biosciences) positive dead cells were excluded for live cell sorting/analysis. Fluorescence labelled single cells were then processed on flow cytometer (Beckman Coutler CytoFLEX) and FACS data were further analyzed with the FlowJo software (Tree star).

\section{Western blotting}

The islets were isolated at the indicated time points. Protein was extracted using radioimmunoprecipitation assay (RIPA) buffer (ThermoFisher) during a 5-minute pipette up-anddown period. 15 micrograms of protein were used for analysis. The following dilutions were used for each antibody: YY1 (Abcam, Cat. No. ab109237, 1:2000), PI3K (Cell Signaling Technology, Cat. No. 4292S, 1:1000), AKT (Cell Signaling Technology, Cat. No. 9272, 1:1000), p-AKT (Ser 473, Cell Signaling Technology, Cat. No. 4060, 1:1000), mTOR (Cell Signaling Technology, Cat. No. 2983, 1:1000), p-mTOR (Ser 2448, Cell Signaling Technology, Cat. No. 5536, 1:1000), S6 (Cell Signaling Technology, Cat. No. 2217, 1:1000), p-S6 (Ser240/244, Cell Signaling Technology, Cat. No. 5364, 1:1000), cytochrome c (Cell Signaling Technology, Cat. No. 4272S, 1:1000), GAPDH (Sigma, Cat. No. G9545, 1:5000) and $\square \square$-actin (ImmunoWay, Cat. No. YM3028, 1:7000). The relative band intensities were quantified using the ImageJ software.

\section{Co-immunoprecipitation assay}

The isolated islets were lysed in lysis buffer A (50 mM HEPES, $10 \mathrm{mM} \mathrm{KCl,} 1 \mathrm{mM}$ EDTA, and $350 \mathrm{mM}$ sucrose) supplemented with proteinase inhibitor (Beyotime) and dithiothreitol (DTT), and incubated on ice for 10 minutes. The lysates were centrifuged for 5 minutes at 2,000 $\mathrm{g}$ and the liquid portion was discarded. The pelleted nuclei were washed three times with lysis buffer A and resuspended in RIPA buffer for 30 minutes at $4^{\circ} \mathrm{C}$, and spun down at $13,200 \mathrm{~g}$ at $4{ }^{\circ} \mathrm{C}$ for 10 minutes. The nuclear supernatants were incubated with IgG (Santa Cruz, sc-2357) or RNA pol II antibody (Active Motif, Cat. No. AB2732926) for overnight with rotation at $4{ }^{\circ} \mathrm{C}$, followed by incubation with Protein-G magnetic beads (Life Technologies, 10006D) for 3 hours with rotation at $4{ }^{\circ} \mathrm{C}$. The immune complex was then washed with RIPA buffer for three times. Bound proteins 
were then eluted with SDS-PAGE sample loading buffer (Beyotime) by incubating at $96^{\circ} \mathrm{C}$ for 10 minutes. The eluted proteins were subjected to western analyses using the indicated antibodies.

\section{RNA sequencing}

RNA-seq and analysis were performed as previously described (16). Total RNA was isolated and analyzed on the Agilent Tape station for RNA integrity numbers (RINs) before library preparation. RNA-seq libraries were prepared using the NEBNext ${ }^{\circledR}$ Ultra $^{\mathrm{TM}}$ II RNA Library Prep Kit (NEB, Cat. No. E7775) according to the manufacturer's protocol. mRNA was isolated using poly$\mathrm{T}$ oligos conjugated to magnetic beads and then fragmented and reverse-transcribed to cDNA. Deoxyuridine triphosphates (dUTPs) were incorporated during second strand synthesis and thus were not amplified. cDNA underwent end-repair, ligation with indexed adapters, and PCR amplification. Nucleic acid was cleaned up after each step using AMPure XP beads (Beckman Coulter). Libraries were quantified, pooled, and sequenced at paired-end $150 \mathrm{bp}$ on the Illumina HiSeq platform with a total of 80 million reads per library.

\section{Quantitative real time RT-qPCR}

Total mRNA was isolated from the purified murine pancreatic islets or cell lines using the RNeasy Mini Kit (QIAGEN) and reverse transcribed using the iScript cDNA Synthesis Kit (Bio-Rad), according to the manufacturers' instructions. Real time reverse transcriptase qPCR was analyzed on the CFX Connect Read-Time PCR Detection system (Bio-Rad) via SYBR Green (Bio-Rad). Gene expression levels were normalized to the housekeeping gene $\square$-actin or Gapdh. The relative gene expression level of each sample was compared with an internal control. Primers used are listed in Table S1.

\section{ChIP sequencing and ChIP-qPCR}

The purified pancreatic islets or cell lines were cross-linked with $1 \%$ formaldehyde at room temperature for 10 minutes. The reaction was quenched by an addition of $0.125 \mathrm{M}$ glycine for 5 minutes, and the cross-linked chromatin was fragmented by sonication (S220, Covaris) for 3 
minutes (Peak power 175W, Duty 10\%, Cycle per burst 200). The sheared chromatin was then diluted by 1:10 with dilution buffer, and 10\% of which was taken to serve as "Input DNA" and negative control. The rest was incubated with $10 \mu \mathrm{YY} 1$ antibody according to the manufacturer's protocol (Active Motif, Cat. No. 61779), and the chromatin/antibody complex was left to rotate end to end at $4{ }^{\circ} \mathrm{C}$ overnight. The reaction was subsequently pulled with protein $\mathrm{G}$ Dynabeads (Thermo Fisher Scientific). The immunoprecipitated genomic DNA was then washed, eluted and reverse cross-linked, followed by RNase and protease treatments. For ChIP-seq, the immunoprecipitated DNA was then resuspended in $20 \mu \mathrm{l}$ of water for library preparation. ChIPseq libraries were prepared according to instructions of the NEBNext Ultra ${ }^{\mathrm{TM}}$ DNA Library Prep Kit for Illumina (NEB, Cat. No. E7645). Sequencing was performed at paired-end 149 bp on the Illumina HiSeq 2500 system according to manufacturer's instructions. For ChIP-qPCR, the precipitated genomic DNA was resuspended in $50 \mu 1$ of water. qPCR was performed with $1 \mu 1$ of immunoprecipitated DNA or input DNA as template via iTaq Universal SYBR Green Supermix (BioRad, Cat No.1725122) on a CFX Connect Real-Time PCR Detection System (BioRad). The relative enrichment values were normalized to the housekeeping gene $\square$ actin. Primers used are listed in Table S2. 
Figure S1 YY1 ablation does not directly alter the cellular composition of pancreatic islets. The following experiments were performed in $P d x 1^{C r e E R T /+} ; Y y l^{f l f l}(\mathrm{cKO})$ and $Y y 1^{f l f l}$ (control) mice at 2 weeks after tamoxifen (Tam) treatment. (A) Immunostaining for glucagon (GCG, red), insulin (INS, green) and somatostatin (SST, yellow) with nuclear DAPI or Hoechst counterstain (blue) on frozen pancreatic sections of cKO and control mice. Scale bars: $100 \mu \mathrm{m}$. (B-F) Quantification of (A) showing no significant difference in (B) $\% \mathrm{GCG}^{+}$cells per islet, (D) $\mathrm{GCG}^{+}$to $\mathrm{INS}^{+}$cell ratio per islet, or (F) $\% \mathrm{SST}^{+}$cells per islet between $\mathrm{cKO}$ and control mice. Significantly reduced (C) $\% \mathrm{INS}^{+}$cells per islet or (E) individual beta cell size in cKO than control mice. (G) RT-qPCR showing the expression levels of genes related to beta cell identity $(P d x 1, N k x 6.1, N g n 3)$ and maturation (Npy, Ucn3) detected in islets isolated from $\mathrm{cKO}$ and control mice. (B-G) Data are presented as mean \pm S.E.M.; $* \mathrm{P}<0.05,{ }^{*} \mathrm{P}<0.01, * * * \mathrm{P}<0.001 ; \mathrm{n}=4-5$ mice per group. 
Fig S1
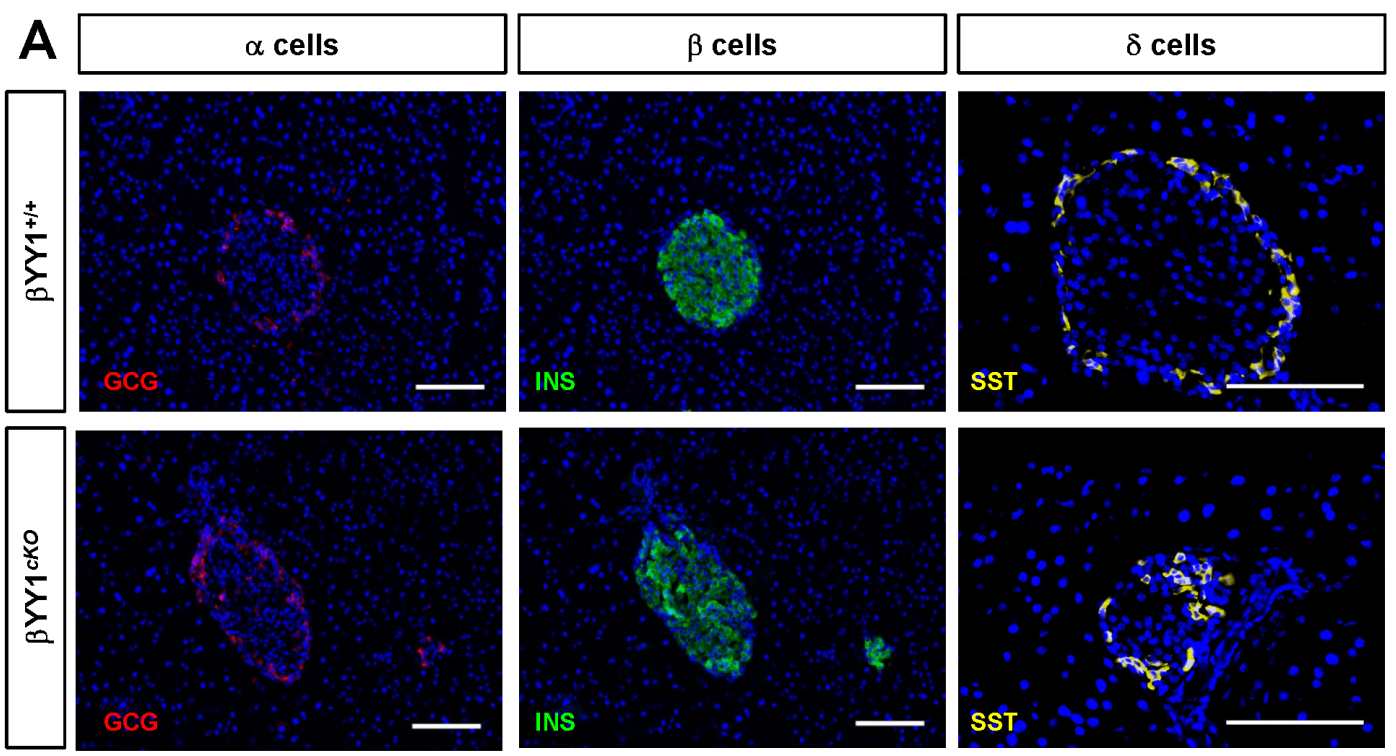

B

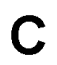

D
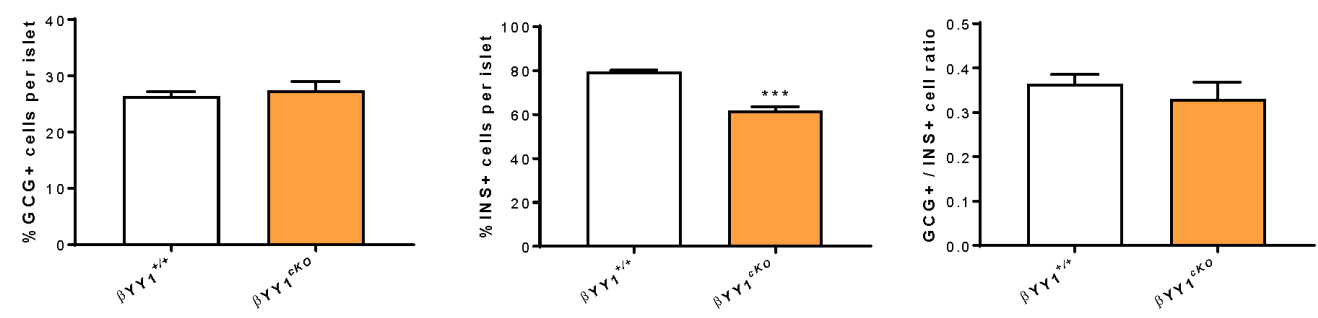

E

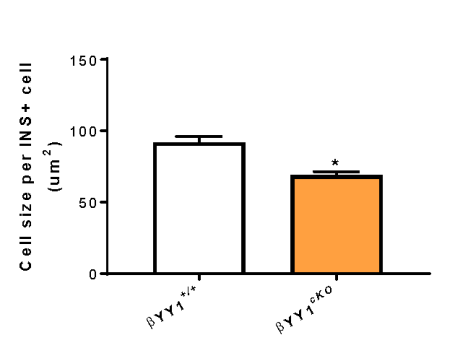

F

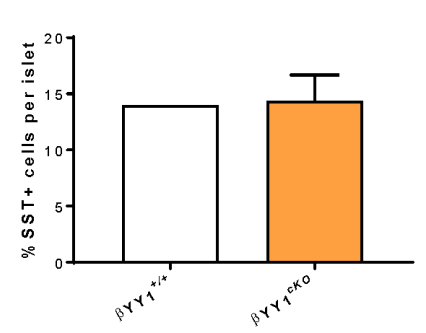

G

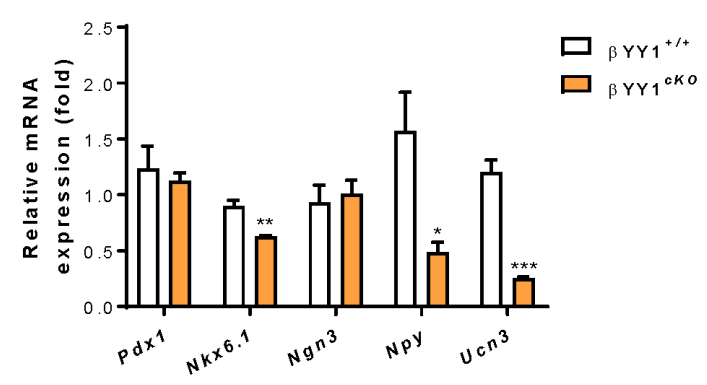


Figure S2 YY1 ablation does not directly contribute to beta cell inflammation. (A) RNA-seq analyses showing the top 5 most significant by GO in terms of biological processes or KEGG pathways determined by upregulated genes expressed by the pancreatic islets of $P d x 1^{\mathrm{CreERT/+}} ; Y y l^{f l f l}$ (cKO) compared to that of $Y y l^{f l f l}$ (control) mice at 2 weeks after tamoxifen (Tam) treatment. (B) RT-qPCR showing the gene expression levels of pro- and anti-inflammatory cytokines detected in islets isolated from $\mathrm{cKO}$ and control mice at 2 weeks after Tam, respectively. (C) Immunostaining for insulin (INS, green) and CD3 (red) with nuclear DAPI or Hoechst counterstain (blue) on frozen pancreatic sections of cKO and control mice at 2 and 12 weeks after Tam, respectively. Scale bars: $100 \mu \mathrm{m}$. (D) Flow cytometric analyses and (E) quantification showing the percentage of $\mathrm{CD}^{+} \mathrm{CD}^{+}$helper $\mathrm{T}$ cells, $\mathrm{CD} 3^{+} \mathrm{CD} 8^{+}$cytotoxic $\mathrm{T}$ cells and $\mathrm{CD} 11 \mathrm{~b}^{+} \mathrm{F} 4 / 80^{+}$macrophages among total $\mathrm{CD} 45^{+}$leukocytes in islets purified from $\mathrm{cKO}$ and control mice, respectively, at 4 weeks after Tam. (B, E-G) Data are presented as mean \pm S.E.M.; $* \mathrm{P}<0.05, * * \mathrm{P}<0.01 ; \mathrm{n}=9$ mice per group. 


\section{A}

GO (Biological Process)

GO:0070098 chemokine-mediated signaling pathway

GO:0006954 inflammatory response

GO:0034097 response to cytokine

\section{KEGG}

mmu04064 NF-kappa B signaling pathway

mmu05340 primary immunodeficiency

mmu04060 cytokine-cytokine receptor interaction

B

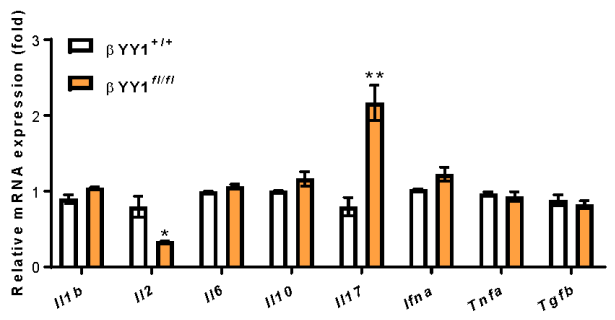

C

Fig S2

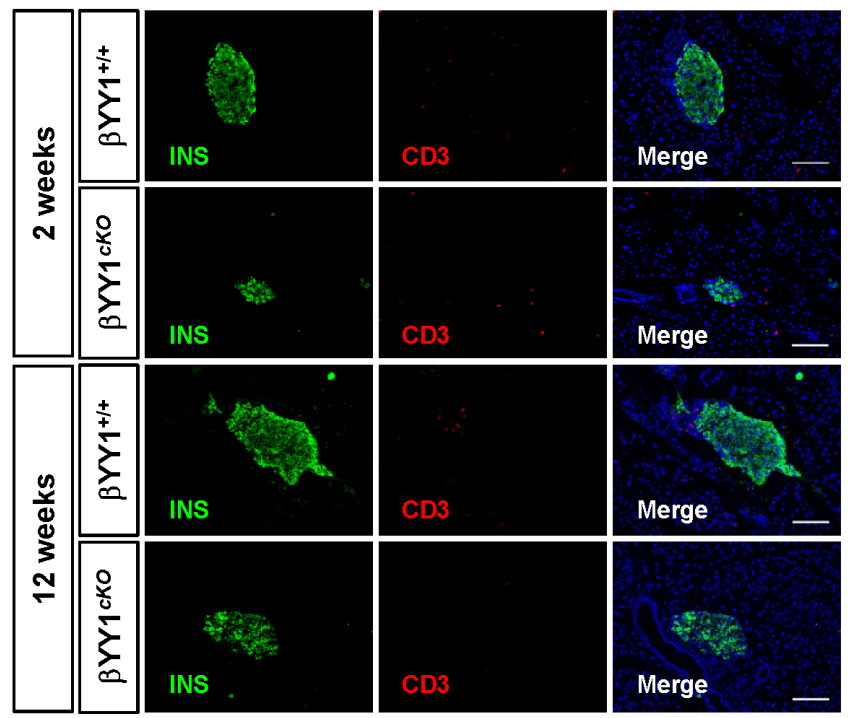

\section{Gated on CD45+}
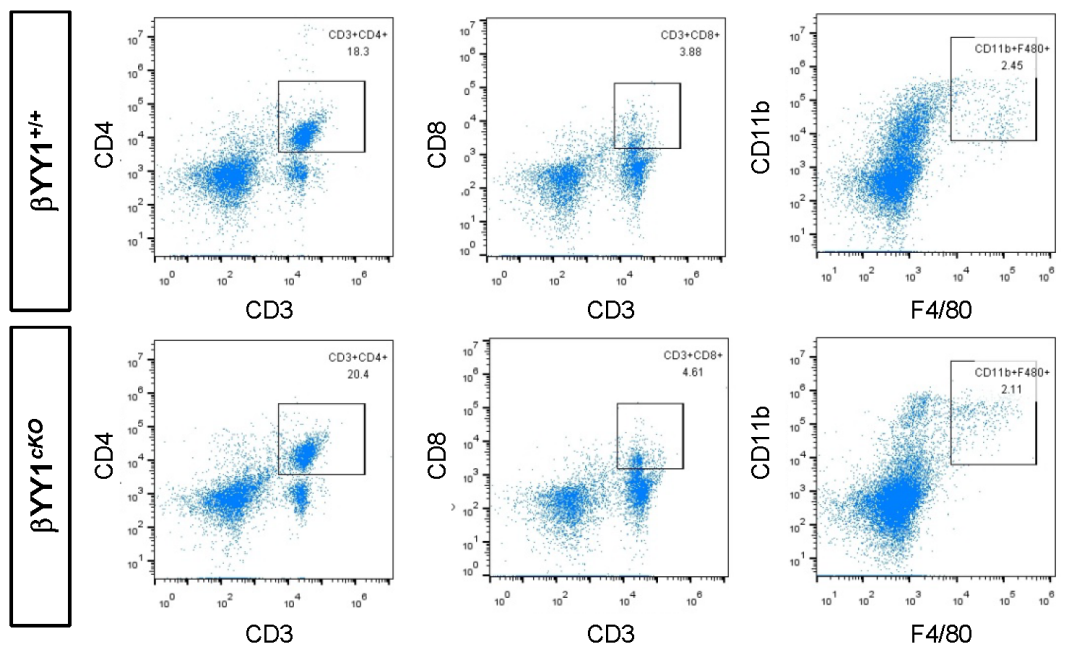

E

F

$\underline{\mathrm{CD} 8+\mathrm{T} \text {-cells }}$
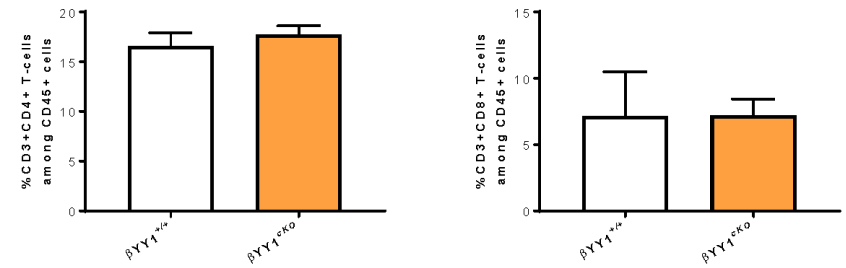

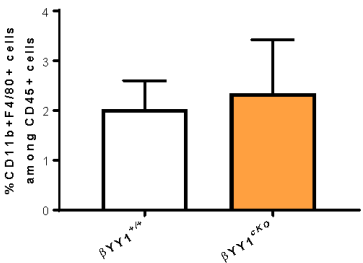

G Macrophages 
Figure S3 YY1 ablation does not directly contribute to beta cell death. The following experiments were performed in $P d x 1^{C r e E R T /+} ; Y y l^{f l f l}$ (cKO) and $Y y l^{f l f l}$ (control) mice at 2 weeks after tamoxifen (Tam) treatment. (A, C) Immunostaining for insulin (INS, green), cleaved caspase 3 (cCASP3, red in A) or phospho(p)- $\gamma \mathrm{H} 2 \mathrm{~A}$.X (red in C) with nuclear DAPI or Hoechst counterstain (blue) on frozen pancreatic sections of $\mathrm{cKO}$ and control mice. Scale bars: $100 \mu \mathrm{m}$. (B) Quantification of (A) showing the number of $\mathrm{cCASP}^{+}$cells per cKO and control islet. (D, E) Western blotting and (F-J) quantification showing (F) significantly reduced expression levels of YY1 but no significant difference in the expression or activity levels of islet proteins including $(G)$ PI3K, (H) p-AKT/AKT, (I) p-mTOR/mTOR and (J) p-rpS6/rpS6 isolated from cKO and control mice. (B, F-J) Data are presented as mean \pm S.E.M.; $* * \mathrm{P}<0.01 ; \mathrm{n}=9$ mice per group; islets of three pancreata were pooled for each band. 
A
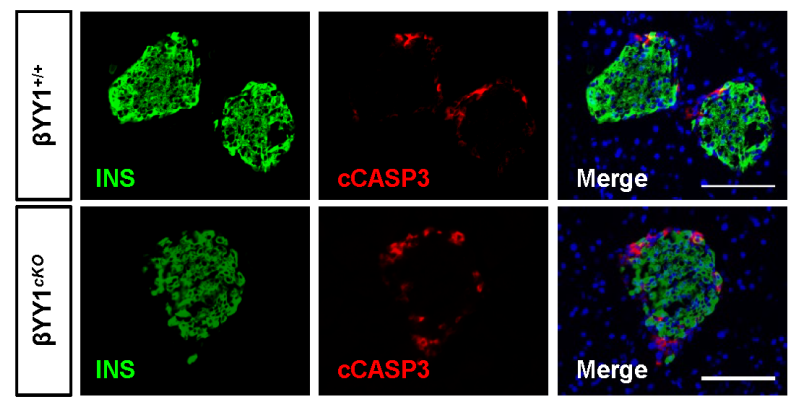

B

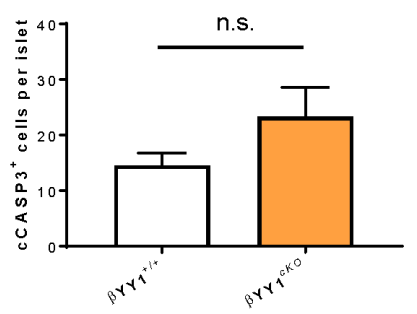

Fig S3

C
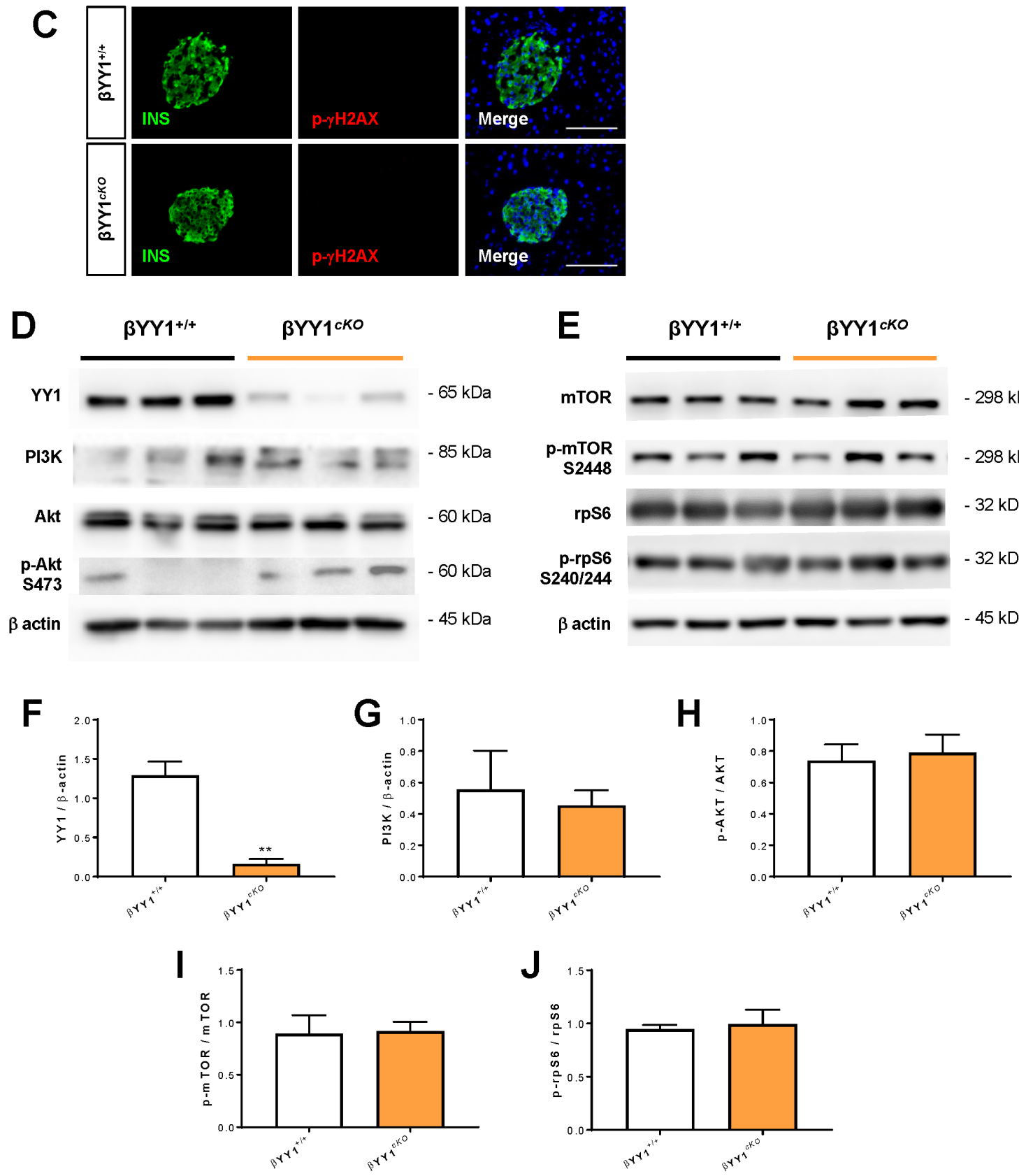
Fig S4 YY1 ablation does not directly contribute to mitochondrial dysfunction in beta cells. (A-C) The following experiments were performed in $P d x 1^{C r e E R T /+} ; Y y 1^{f l f l}$ (cKO) and $Y y 1^{f l f l}$ (control) mice at 2 weeks after tamoxifen (Tam) treatment. (A) Immunostaining and quantification for insulin (INS, green) and MitoSOX red (red) with nuclear DAPI or Hoechst counterstain (blue) on living cells isolated from $\mathrm{cKO}$ and control mice. Scale bars: $10 \mu \mathrm{m}$. (B) Immunostaining and quantification for insulin (INS, green) and TMRE (red) with nuclear DAPI or Hoechst counterstain (blue) on living cells isolated from cKO and control mice. Scale bars: $10 \mu \mathrm{m}$. (C) Flow cytometric analyses and quantification showing no significant difference in $\% \mathrm{TMRE}^{+}$cells in $\mathrm{cKO}$ (red peak) and control (blue peak) islets. (D) RT-qPCR showing the gene expression levels of cytochrome c (Cyto $\mathrm{C}$ ) detected in islets isolated from $\mathrm{cKO}$ and control mice at 4 days and 2 weeks after Tam, respectively. (E) Western blotting and quantification showing significantly reduced expression levels of cytochrome $\mathrm{c}$ isolated from $\mathrm{cKO}$ than that of the control islets at 6 weeks after Tam. Islets of three pancreata were pooled for each band. (A-E) Data are presented as mean \pm S.E.M.; $*$ P $<0.05$; $\mathrm{n}=4-5$ mice per group. 
Fig S4
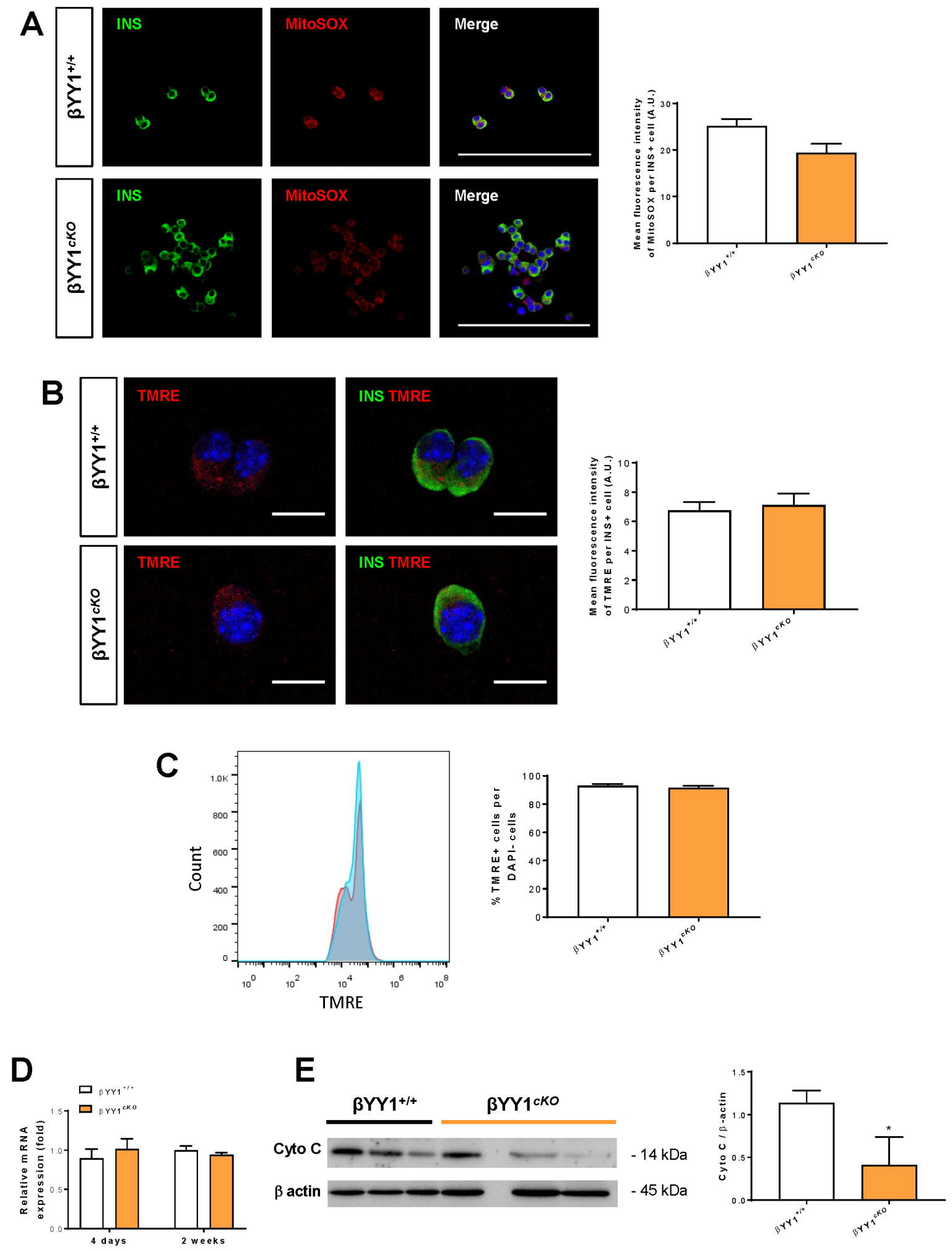
Figure S5 Genome-wide ChIP-seq analyses showing direct binding sites of YY1 in Rims2, Pdpk1 and Ins1. Genome snapshots for YY1 ChIP-seq analyses performed in pancreatic islets covering the differentially expressed genes identified by bulk RNA-seq in Figure 4D-G and by RT-qPCR in Figure S1G, respectively. Green boxes denote the YY1 binding peaks. 
Fig S5

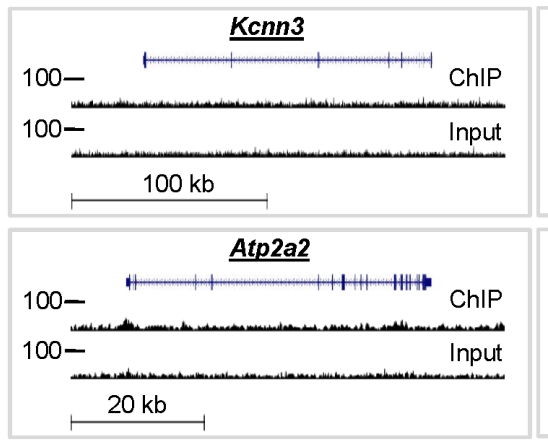

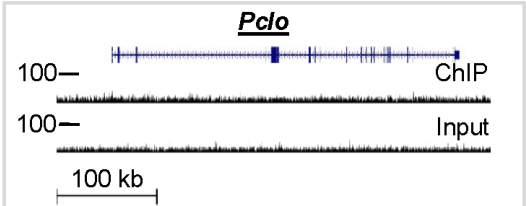
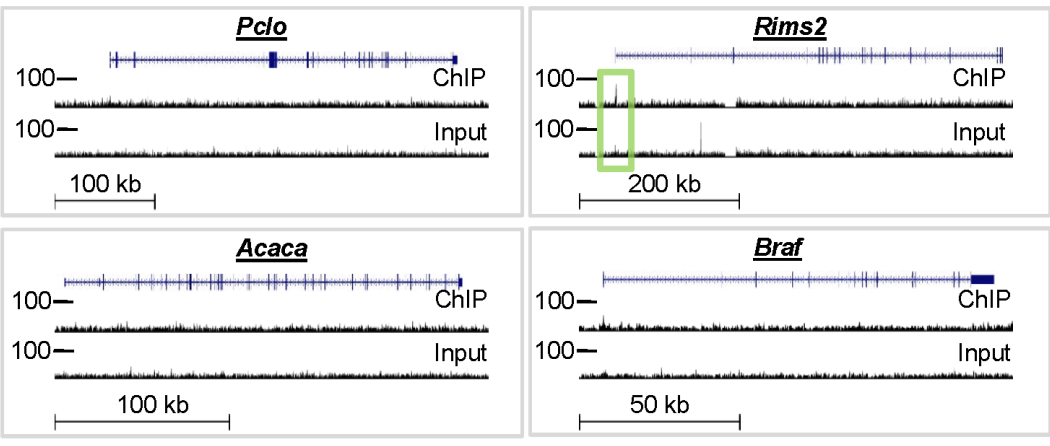

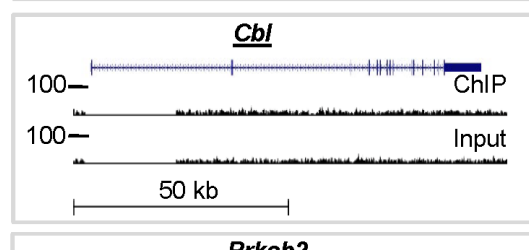

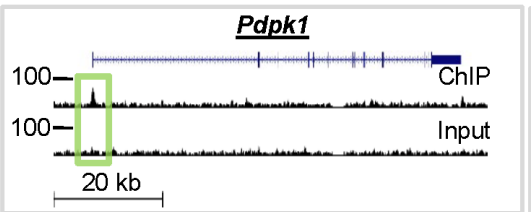
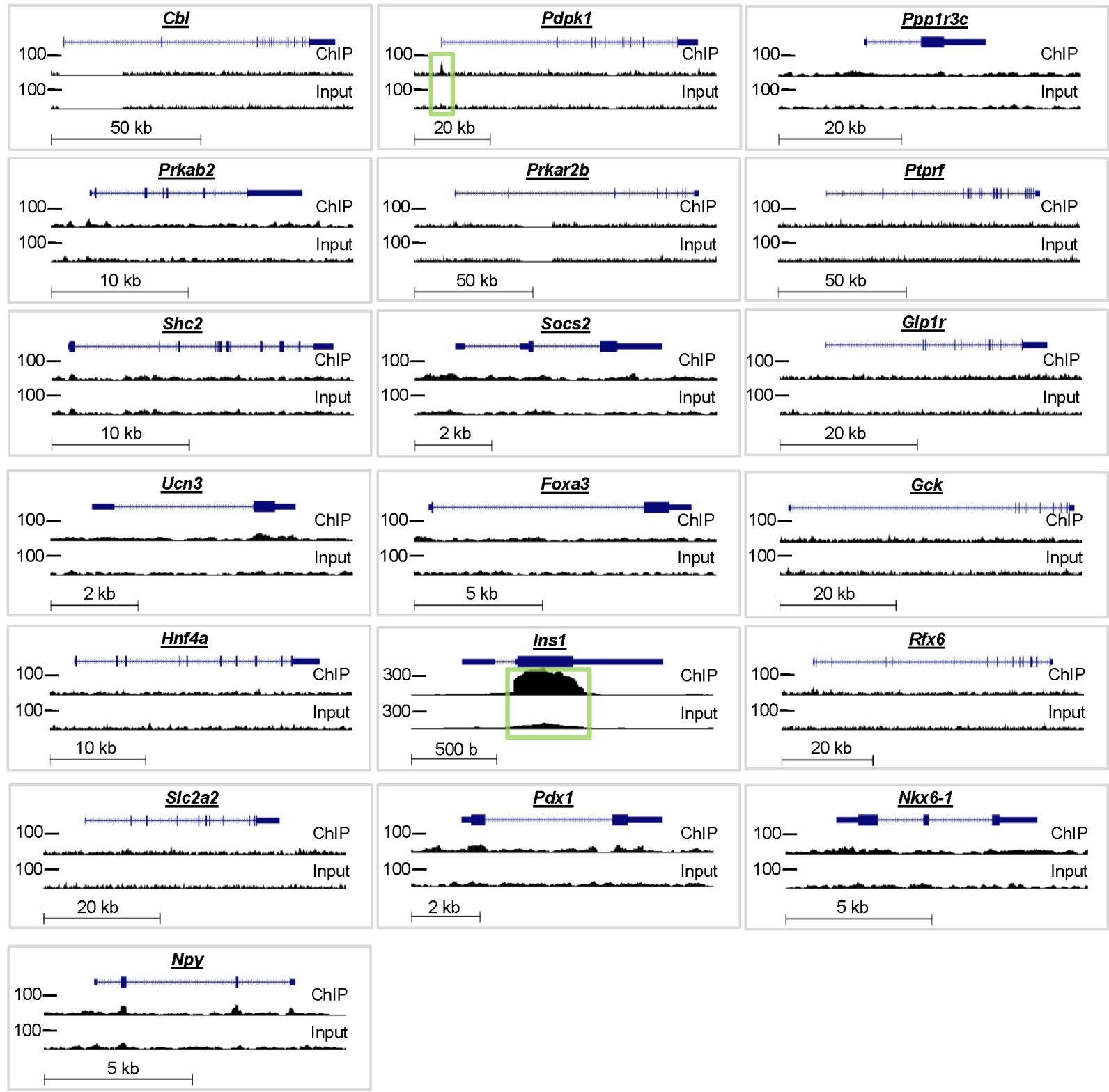
Figure S6 The canonical binding sites of YY1 in enhancer regions of the insulin genes are conserved across mouse and rat. Pairwise alignment of mouse and rat YY1 binding regions on exon 2 of the (A) Ins1 and (B) Ins2 genes was determined by Clustal Omega. Asterisks (*) denote identical nucleotides between the two DNA sequences. Blue boxes indicate the core motif sequence of YY1.

Fig S6

\section{A}

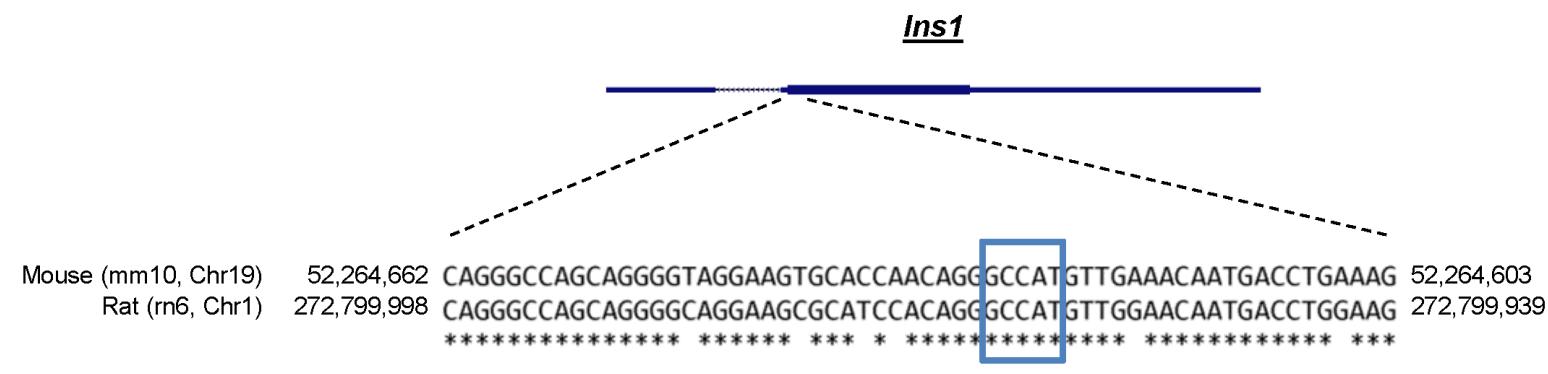

B

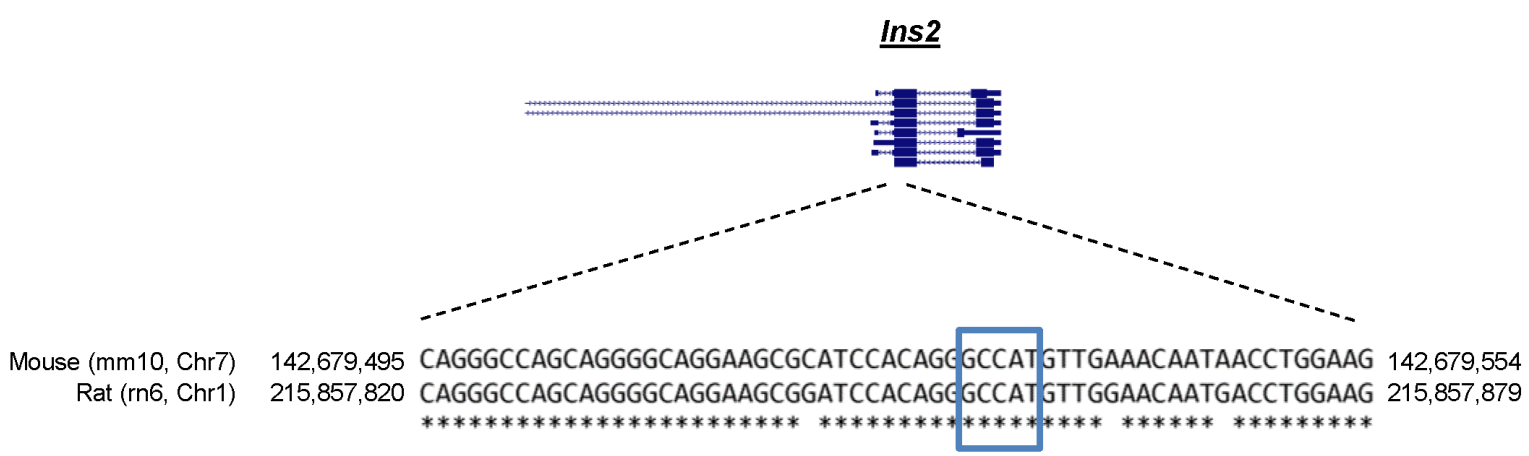


Table S1 Quantitative RT-qPCR primers used in this study.

\begin{tabular}{|c|c|c|}
\hline $\begin{array}{l}\text { Mouse } \\
\text { Genes }\end{array}$ & Forward & Reverse \\
\hline Yy1 & CCTCTACATCGCCACGGAC & TGGTCTCCACCTCGATCTCA \\
\hline Ins 1 & CGTGGCTTCTTCTACACACCCA & TGCAGCACTGATCCACAATGCC \\
\hline Ins2 & CGTGGCTTCTTCTACACACCCA & TCCAGTGCCAAGGTCTGAAGGT \\
\hline Kcnn3 & TCCACCGTCATCCTGCTTGGTT & CAGGCTGATGTAGAGGATACGC \\
\hline Pclo & $\begin{array}{c}\text { TCTGGAGGAACTGAAGCAGCA } \\
\text { G }\end{array}$ & CAACTCCCGAAATGACTTGGCG \\
\hline Rims2 & GATGTCTCCTCATTGCCACTCC & ATCCTCGCAGTCGTAGTCAGAC \\
\hline Alp2a2 & $\begin{array}{c}\text { GTGAAGTGCCATCAGTATGACG } \\
\text { G }\end{array}$ & GTGAGAGCAGTCTCGGTAGCTT \\
\hline Acaca & $\begin{array}{c}\text { GTTCTGTTGGACAACGCCTTCA } \\
\text { C }\end{array}$ & GGAGTCACAGAAGCAGCCCATT \\
\hline Braf & $\begin{array}{l}\text { CGCCAAGTCAATCATCCACAGA } \\
\text { G }\end{array}$ & CACCGAGATTTCACTGTGGCTAG \\
\hline $\mathrm{Cbl}$ & TGATGAAGGAGTTGGCAGGTGC & TGAAGTGCTGGCAGGAACAGGT \\
\hline Pdpk1 & CTACCAGCCATGTCAGAGGATG & AGGCTGGTTTCCACCGTAGACA \\
\hline $\begin{array}{l}\text { Ppp1r3 } \\
\text { c }\end{array}$ & TGCTTGGCTCATTCACCACCTC & CACTCTGCGATTTGGCTTCCTG \\
\hline Prkab2 & GACTTCGTTGCCATCCTGGATC & CCAAGCTGACTGGTAACCACAG \\
\hline $\begin{array}{l}\text { Prkar2 } \\
\text { b }\end{array}$ & CCAGTAAGGGTGTCAACTTCGC & ACCGAGGCACGCCTTGTGAAC \\
\hline Ptprf & CAGATTCGTGGCTACCAGGTCA & ACGGTGATGGAGTAGGTGGTCT \\
\hline Shc2 & GCAGTCTATTTCCTTCGCCTCG & GGCAAGACCTTCACAGCATTCC \\
\hline Socs2 & GCGCGTCTGGCGAAAGCCCT & GAAAGTTCCTTCTGGAGCCTCTT \\
\hline Glp1r & CGGAGTGTGAAGAGTCTAAGCG & ATGGCTGAAGCGATGACCAAGG \\
\hline Ucn3 & $\begin{array}{c}\text { ACAACCAAACGCACCTCCAGAT } \\
\text { C }\end{array}$ & TTGTGCTGTGCCTGGGATTGGT \\
\hline Foxa3 & GTCCACACCTTATTTCAGCGGC & CACATCCAGTTTGGAAGGTGTCG \\
\hline Gck & $\begin{array}{l}\text { GCATCTCTGACTTCCTGGACAA } \\
\text { G }\end{array}$ & CTTGGTCCAGTTGAGCAGGATG \\
\hline
\end{tabular}




\begin{tabular}{|c|c|c|}
\hline Hnf4a & TGCGAACTCCTTCTGGATGACC & CAGCACGTCCTTAAACACCATGG \\
\hline Rfx6 & GCACCCATTCTCAACTGAGGAC & CTATGGCTGGAGAAATCGGTGG \\
\hline Slc2a2 & $\begin{array}{c}\text { GTTGGAAGAGGAAGTCAGGGC } \\
\text { A }\end{array}$ & ATCACGGAGACCTTCTGCTCAG \\
\hline $\mathrm{Il} 1 \mathrm{~b}$ & TGGACCTTCCAGGATGAGGACA & $\begin{array}{c}\text { GTTCATCTCGGAGCCTGTAG } \\
\text { TG }\end{array}$ \\
\hline $\mathrm{I} 12$ & CAAGCAGGCCACAGAATTGA & CTCAAATGTGTTGTCAGAGCCC \\
\hline I16 & TACCACTTCACAAGTCGGAGGC & CTGCAAGTGCATCATCGTTGTTC \\
\hline Il10 & CGGGAAGACAATAACTGCACCC & CGGTTAGCAGTATGTTGTCCAGC \\
\hline Il17 & GGCCCTCAGACTACCTCAAC & TCTCGACCCTGAAAGTGAAGG \\
\hline Ifn $\alpha$ & AGCTCTGCATCGTTTTGGGT & TTCTGTCACTCTCCTCTTTCCA \\
\hline $\operatorname{Tnf} \alpha$ & CCTGTAGCCCACGTCGTAG & GGGAGTAGACAAGGTACAACCC \\
\hline $\operatorname{Tgf} \beta$ & $\begin{array}{c}\text { TTTGGAGCCTGGACACACAGTA } \\
\text { CA }\end{array}$ & TGTGTTGGTTGTAGAGGGCAAGGA \\
\hline$\beta$ actin & $\begin{array}{c}\text { CATTGCTGACAGGATGCAGAAG } \\
\text { G }\end{array}$ & TGCTGGAAGGTGGACAGTGAGG \\
\hline
\end{tabular}


Table S2 Quantitative real time ChIP-qPCR primers used in this study.

\begin{tabular}{|c|c|c|}
\hline Mouse Genes & Forward & Reverse \\
\hline Ins1 & GTGCACTTCCTACCCCTGCT & GTAGAAGAAGCCACGCTCCC \\
\hline Ins2 & TGCTATCCTCAACCCAGCCTA & GGACTCCCAGAGGAAGAGC \\
\hline Yy1 & CATGTGGTCCTCGGGTGAG & GGGCGGAGAAGATGGCG \\
\hline$\beta$ actin & GCGCAAGTACTCTGTGTGGA & CGGACTCATCGTACTCCTGC \\
\hline
\end{tabular}


Table S3 GO enrichment analyses showing a list of genes expressed in the islets of cKO compared to that of control mice at 2 weeks after Tam treatment. The top 5 pathways in terms of biological processes that were most significantly downregulated were determined by genome-wide transcriptome profiling, related to Figure 4.

\begin{tabular}{|l|l|l|}
\hline Pathway & P-value & Genes \\
\hline $\begin{array}{l}\text { GO:0009749 response to } \\
\text { glucose }\end{array}$ & $6.87303 \mathrm{E}-07$ & $\begin{array}{l}\text { LPL, SLC8A1, PFKFB2, NNAT, } \\
\text { MLXIPL, FKBP1B, TCF7L2, ACVR1C, } \\
\text { UCN3, ACVR2B, GLUL, HNF4A, } \\
\text { GIPR, SLC30A8, GLP1R }\end{array}$ \\
\hline $\begin{array}{l}\text { GO:0032024 positive } \\
\text { regulation of insulin secretion }\end{array}$ & $1.31548 \mathrm{E}-06$ & $\begin{array}{l}\text { GLUL, UCN3, GCK, PFKFB2, GPR39, } \\
\text { NNAT, GIPR, FFAR1, ABAT, SLC30A8, } \\
\text { TCF7L2, GLP1R }\end{array}$ \\
\hline $\begin{array}{l}\text { GO:0030073 insulin } \\
\text { secretion }\end{array}$ & $3.04405 E-06$ & $\begin{array}{l}\text { ACVR2B, GPR119, HTT, FFAR1, } \\
\text { RAPGEF4, SLC30A8, RIMS2, } \\
\text { FKBP1B, PCLO, GLP1R }\end{array}$ \\
\hline $\begin{array}{l}\text { GO:0017157 regulation of } \\
\text { exocytosis }\end{array}$ & 0.001774011 & $\begin{array}{l}\text { RAB3B, RPH3AL, RAPGEF4, RIMS2, } \\
\text { PCLO, NSF }\end{array}$ \\
\hline $\begin{array}{l}\text { GO:0031018 endocrine } \\
\text { pancreas development }\end{array}$ & 0.003343676 & $\begin{array}{l}\text { HNF4A, GCK, FOXA3, RFX6, } \\
\text { SLC2A2, GIPR, INS1 }\end{array}$ \\
\hline
\end{tabular}


Table S4 KEGG analyses showing a list of genes expressed in the islets of cKO compared to that of control mice at 2 weeks after Tam treatment. The top 5 pathways in terms of KEGG pathways that were most significantly downregulated were determined by genomewide transcriptome profiling, related to Figure 4.

\begin{tabular}{|l|l|l|}
\hline Pathway & P-value & Genes \\
\hline mmu04911: insulin secretion & $4.06328 \mathrm{E}-09$ & $\begin{array}{l}\text { KCNMA1, PRKCA, GPR119, ATP1B2, } \\
\text { FFAR1, ITPR3, RIMS2, PCLO, } \\
\text { PRKCB, KCNMB2, GCK, ADCY9, } \\
\text { KCNN3, SLC2A2, CAMK2B, } \\
\text { RAPGEF4, INS1, GLP1R }\end{array}$ \\
\hline $\begin{array}{l}\text { mmu04919: thyroid hormone } \\
\text { signaling pathway }\end{array}$ & $3.50718 \mathrm{E}-05$ & $\begin{array}{l}\text { PRKCA, THRB, ATP1B2, PFKFB2, } \\
\text { MED12, MED13, RCAN2, PRKCB, } \\
\text { MED12L, CCND1, PDPK1, NCOA1, } \\
\text { NCOA2, DIO1, NCOR1 }\end{array}$ \\
\hline $\begin{array}{l}\text { mmu04950: maturity onset } \\
\text { diabetes of the young }\end{array}$ & 0.002058791 & $\begin{array}{l}\text { HNF4A, GCK, FOXA3, RFX6, } \\
\text { SLC2A2, INS1 }\end{array}$ \\
\hline $\begin{array}{l}\text { mmu04972: pancreatic } \\
\text { secretion }\end{array}$ & 0.002306006 & $\begin{array}{l}\text { KCNMA1, PRKCA, ADCY9, ATP2A2, } \\
\text { ATP1B2, ITPR3, CPB2, ITPR1, } \\
\text { PRKCB, PLA2G2F, ITPR2 }\end{array}$ \\
\hline $\begin{array}{l}\text { mmu04910: insulin signaling } \\
\text { pathway }\end{array}$ & 0.003251078 & $\begin{array}{l}\text { PTPRF, BRAF, SOCS2, PRKAB2, CBL, } \\
\text { ACACA, PPARGC1A, PRKAR2B, } \\
\text { PPP1R3C, PDPK1, GCK, INS1, SHC2 }\end{array}$ \\
\hline
\end{tabular}


Table S5 GO enrichment analyses showing a list of genes expressed in the islets of cKO compared to that of control mice at 2 weeks after Tam treatment. The top 3 pathways in terms of biological processes that were most significantly upregulated were determined by genome-wide transcriptome profiling, related to Figure S1.

\begin{tabular}{|c|c|c|}
\hline Pathway & P-value & Genes \\
\hline $\begin{array}{l}\text { GO:0070098 chemokine- } \\
\text { mediated signaling pathway }\end{array}$ & $3.60744 \mathrm{E}-09$ & $\begin{array}{l}\text { CXCL1, CCL3, CXCL5, CCL21C, } \\
\text { CXCL2, CCL21A, ACKR1, CCL21B, } \\
\text { CCL19, CCL5, CXCL12, CCL11, } \\
\text { CCL25, CCL22, GM10591 }\end{array}$ \\
\hline $\begin{array}{l}\text { GO:0006954 inflammatory } \\
\text { response }\end{array}$ & 1.26169E-08 & $\begin{array}{l}\text { CXCL1, HMGB2, CCL3, TNF, PTGS2, } \\
\text { CXCL5, C3, CXCL2, CCL5, CXCL12, } \\
\text { TNFRSF4, CCL25, CCL22, CYP26B1, } \\
\text { IL1B, BCL6, SPP1, BMP2, C4B, } \\
\text { CCL21C, RELB, ACKR1, CCL21A, } \\
\text { CCL21B, CCL19, CHST4, CD4, } \\
\text { CCL11, PRKCQ, LAT, CYBA, CCR7, } \\
\text { GM10591, TNFAIP3, SELE, IGFBP4 }\end{array}$ \\
\hline $\begin{array}{l}\text { GO:0034097 response to } \\
\text { cytokine }\end{array}$ & $1.02102 \mathrm{E}-07$ & $\begin{array}{l}\text { PTGS2, COL3A1, RELB, } \text { ACP5, } \\
\text { SERPINA1E, CCL5, JUNB, TIMP1, } \\
\text { FOS, SERPINA3N, } \text { CORO1A, } \\
\text { SERPINA1B, JUN, SERPINA1D, } \\
\text { JUND, SERPINA1C }\end{array}$ \\
\hline
\end{tabular}


Table S6 KEGG analyses showing a list of genes expressed in the islets of cKO compared to that of control mice at 2 weeks after Tam treatment. The top 3 pathways in terms of KEGG pathways that were most significantly upregulated were determined by genome-wide transcriptome profiling, related to Figure S1.

\begin{tabular}{|l|l|l|}
\hline Pathway & P-value & Genes \\
\hline $\begin{array}{l}\text { mmu04064:NF-kappa B } \\
\text { signaling pathway }\end{array}$ & $3.06422 \mathrm{E}-07$ & $\begin{array}{l}\text { TNF, PTGS2, CCL21C, RELB, } \\
\text { CCL21A, NFKBIA, CCL21B, CCL19, } \\
\text { CD40, CXCL12, VCAM1, LAT, } \\
\text { PRKCQ, BCL2A1D, GM10591, IL1B, } \\
\text { TNFAIP3, LTB }\end{array}$ \\
\hline $\begin{array}{l}\text { mmu05340:primary } \\
\text { immunodeficiency } \\
\text { mmu04060:cytokine-cytokine }\end{array}$ & $1.63611 \mathrm{E}-05$ & $\begin{array}{l}\text { CXCL1, IL1R2, CCL3, TNF, CXCL5, } \\
\text { CSF2RB2, CXCL2, CCL5, IL7R, } \\
\text { TNFRSF4, CXCL12, CCL25, CCL22, } \\
\text { IL1B, LTB, BMP2, IL2RB, IL7, } \\
\text { CCL21C, CCL21A, CCL19, CCL21B, } \\
\text { CD40, CCL11, CCR7, GM10591 }\end{array}$ \\
\hline
\end{tabular}


Table S7 De novo motif discovery showing the top motif within peaks identified by YY1 ChIP-Seq analysis on islet, related to Figure 5.

\begin{tabular}{|l|l|}
\hline Item & Value \\
\hline Rank & 1 \\
\hline Motif & $1 \mathrm{E}-378$ \\
\hline P-value & $-8.724 \mathrm{e}+02$ \\
\hline log P-value & $69.17 \%$ \\
\hline$\%$ of Peaks & $3.47 \%$ \\
\hline$\%$ of Background & $48.8 \mathrm{bp}(72.3 \mathrm{bp})$ \\
\hline STD (Bg STD) & YY1/MA0095.2/Jaspar \\
\hline Best Match &
\end{tabular}

